\title{
Delayed Footdrop After Total Hip Arthroplasty: Spinal Stenosis the Cause?
}

\author{
MM Marican, MRCS, IS Rikhraj, FRCS \\ Department of Orthopaedic Surgery, Singapore General Hospital, Singapore
}

\begin{abstract}
Degenerative joint disease of the lower limbs and degenerative lumbar stenosis are commonly seen in the elderly population. Although hip arthroplasty is routinely performed, it is not without complications. We present a case of a 64-year-old lady with arthritic hip pain and asymptomatic lumbar spine stenosis. This patient underwent a primary total hip replacement for hip arthritis and developed an ipsilateral foot drop one day after surgery. A hip MRI showed likely sciatic nerve impingement over a tense capsule. Despite revision surgery, the foot drop persisted. Nerve conduction studies and a lumbosacral MRI revealed spinal canal stenosis at $\mathrm{L} 4 / 5$. When nerve palsies such as a foot drop occur after hip arthroplasty, spinal stenosis must be considered as part of differential diagnosis and should be radiologically excluded before performing exploratory surgery.
\end{abstract}

Key Words:

Hip Arthroplasty, Foot Drop, Spinal Stenosis

\section{INTRODUCTION}

Degenerative joint diseases of the hip and lumbar spinal stenosis are conditions commonly seen in the elderly population and may coexist. Although hip arthroplasty is routinely performed to relieve pain and improve quality of life, it is not without complications. Surgical complications such as wound infection, implant failure and sciatic nerve injury are associated with significant morbidity for this procedure. Foot drop after hip arthroplasty has been reported in the literature and is often due to mechanical impingement of either the implant or a capsular haematoma. We report here a case of an elderly patient who developed foot drop one day after total hip replacement surgery and presented a diagnostic dilemma regarding the cause of this postoperative complication. We explore the association of this apparent postoperative complication with previously undiagnosed spinal stenosis in our patient.

Our patient was a 64 year old lady with progressive mechanical hip pain over the past year, which was clinically and radiologically diagnosed as hip osteoarthritis. Significantly she had no back pain or lower limb neurological abnormalities. She underwent an uneventful left total hip replacement and was recovering with nursing care that included an abduction pillow. When assessed several hours later on the ward, the patient had full range of movement of her left foot and left toes with normal sensation throughout. One day after surgery, she was found to have foot drop on the operative side and numbness over the dorsomedial aspect of her left foot. Initial radiographs did not reveal any hip dislocation and an MRI of her hip showed that the posterior-most acetabular screw was immediately adjacent and inferior to the sciatic nerve as it exited the greater sciatic notch.

Postulating that the foot drop might have been due to the acetabular screw impinging on the sciatic nerve, the patient underwent revision hip surgery. Intraoperatively, the sciatic nerve appeared tense and tented over the capsule. The capsular haematoma was drained and implants were removed. Care was taken to ensure no nerve impingement after the new implants were inserted. A physical examination of her left foot after revision surgery failed to show any clinical improvement in the foot drop and the patient was placed in an ankle-foot orthosis (AFO).

A subsequent motor nerve conduction study showed reduced compound muscle action potentials (CMAPs) of the left tibial and peroneal nerves (L4/5) while the sensory study showed absent sensory nerve action potentials (SNAPs) of the left peroneal and sural nerves in keeping with the diagnosis of L4/5 radiculopathy. A lumbosacral MRI revealed moderate spinal canal and severe bilateral lateral recess with foraminal canal stenosis at $L 4 / 5$ caused by a combination of a disc bulge and facet joint and ligamentum flavum hypertrophy. Decompression surgery was offered to the patient but she opted for conservative management. Despite recovering some sensation over the tibialis anterior and big toe over the next two years and some active dorsiflexion of the big toe, the patient continued to have a foot drop.

\section{DISCUSSION}

Significant lumbar spinal stenosis and lower limb arthritis may co-exist in the elderly population. However, the 


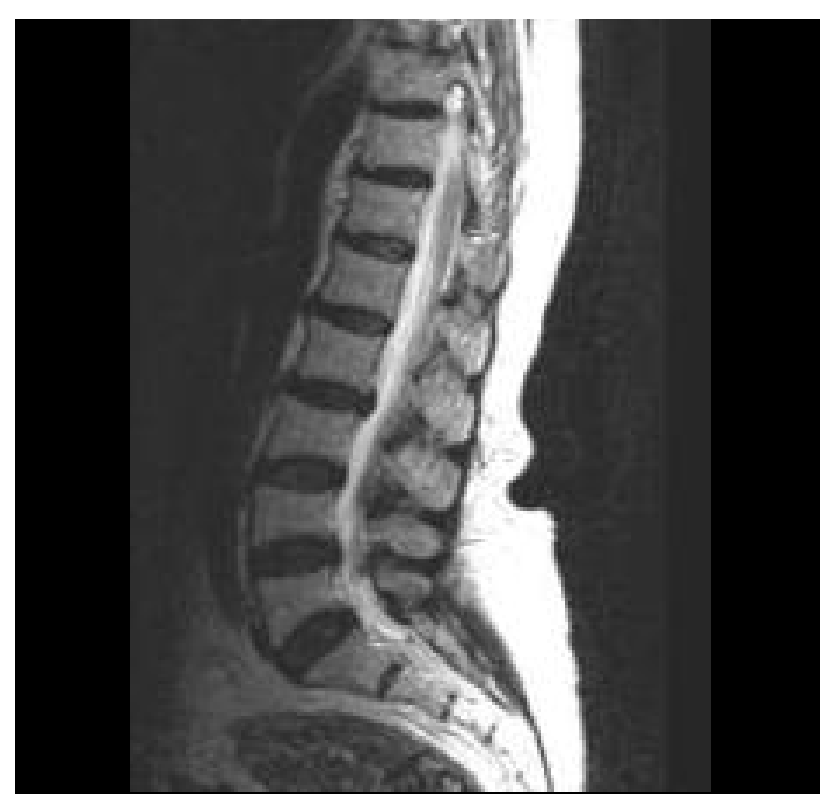

Fig. 1: $\quad$ MRI showing spinal stenosis at $L 3 / 4$ and $L 4 / 5$.

problem lies in patients who have symptomatic lower limb arthritis but asymptomatic spinal stenosis. The incidence of asymptomatic spinal stenosis in patients aged above 60 years old may be as high as $60 \%{ }^{1}$. Considering the difficulty in diagnosing asymptomatic spinal stenosis, surgeons planning to perform lower limb arthroplasty for their older patients ought to actively exclude spinal stenosis with a more detailed history and physical examination regarding neurogenic claudication and radiculopathy. Although lumbar spine xrays are known to correlate poorly with spinal stenosis, it is not cost effective for all patients above 60 years old undergoing lower limb arthroplasty surgery to have a lumbosacral MRI scan. If however the patient has an MRI proven diagnosis of spinal canal stenosis, they should be counselled about the possibility of undergoing surgeries to treat both the lower limb arthritis and spinal canal stenosis. They should also be told of the increased risk of neurological symptoms after lower limb arthroplasty due to the spinal stenosis. There is however no clinical evidence to support spinal decompression in patients who have asymptomatic spinal stenosis.

Large nerves such as the sciatic nerve are prone to injury during significant manipulation involved in total hip surgery. Hip arthroplasty is associated with a $0.6 \%$ to $1.3 \%$ risk of

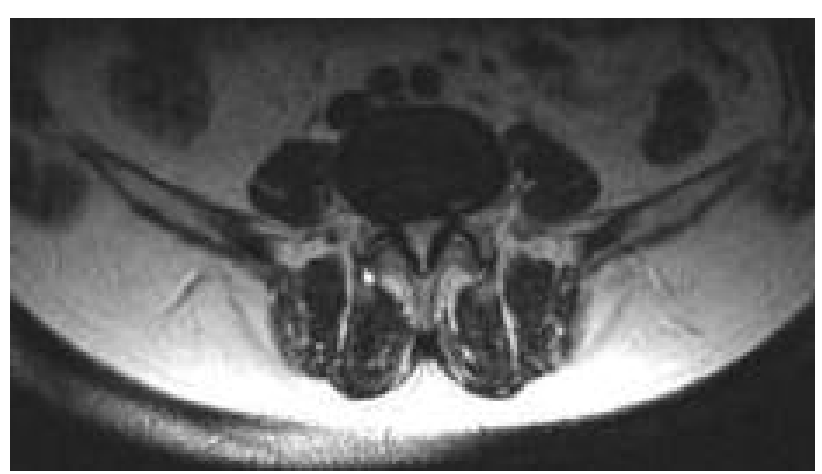

Fig. 2: Axial MRI cut of $L 4 / 5$ degenerate disc with spinal stenosis.

nerve injury but these rates increase to about 3 to $6 \%$ after revision hip surgery ${ }^{2}$. Risk factors for nerve injury associated with hip arthroplasty include diabetes, surgical trauma, hip dislocation as well as alcoholic and nutritional neuropathy. Patients with these underlying co-morbidities have subclinically diseased neurons ${ }^{3}$. The trauma of hip surgery then imposes a moderate crush injury to the diseased neuron potentially leading to symptomatic foot drop. Some patients however develop subclinical nerve injury after surgery; studies have shown that up to $70 \%$ of patients with EMG proven nerve injury after hip arthroplasty are asymptomatic ${ }^{3}$.

With the 'double crush injury hypothesis', a patient with lumbar stenosis alone does not produce a sufficient reduction in neuronal axoplasmic flow to cause clinical signs; however a double injury at the spine and hip impedes neuronal axoplasmic flow enough to cause foot drop ${ }^{4}$. The concept of serial constraints to axoplasmic flow explains why a single crush injury to a diseased neuron would be symptomatic while a moderate crush to a normal neuron is likely to be asymptomatic $^{5}$. Once the underlying cause of the foot drop has been identified, most patients do well with decompression surgery ${ }^{5}$.

\section{CONCLUSIONS}

When nerve palsies such as foot drop occur after hip arthroplasty, spinal stenosis should be considered as one of the differential diagnoses and radiologically excluded before exploratory surgery. 


\section{REFERENCES}

1. Borenstein DG, O’ Mara JW Jr, Boden SD, Lauerman WC, Jacobson A, Platenberg C, et al. The value of magnetic resonance imaging of the lumbar spine to predict low-back pain in asymptomatic subjects: a seven-year follow-up study. J Bone Joint Surg Am 2001;8 3-A(9): 1306-11.

2. McNamara MJ, Barrett KG, Christie MJ, Spengler DM. Lumbar spinal stenosis and lower extremity arthroplasty. J Arthroplasty 1993; 8(3): 273-7.

3. Weber ER, Daube JR, Coventry MB. Peripheral neuropathies associated with total hip arthroplasty. J Bone Joint Surg 1976; 58A: 66.

4. Upton AR, McComas AJ. The double crush and nerve entrapment syndromes. Lancet 1973; 2: 359.

5. Pritchett JW. Lumbar Decompression to Treat Foot Drop After Hip Arthroplasty. Clin Orthop Relat Res 1994; (303): 173-7. 